\title{
NMR Detection of Bifurcated Hydrogen Bonds in Large Proteins
}

Aizhuo Liu ${ }^{*},+$, Zhenwei Lu ${ }^{\dagger}$, Jifeng Wang ${ }^{\dagger}$, Lishan Yao ${ }^{\dagger, \neq, 1}$, Yue Li ${ }^{\dagger}$ and Honggao Yan ${ }^{*,+}$ ${ }^{\dagger}$ Department of Biochemistry and Molecular Biology and ${ }^{\ddagger}$ Department of Chemistry, Michigan State University, East Lansing, MI 48824, USA

\section{Supporting Information}




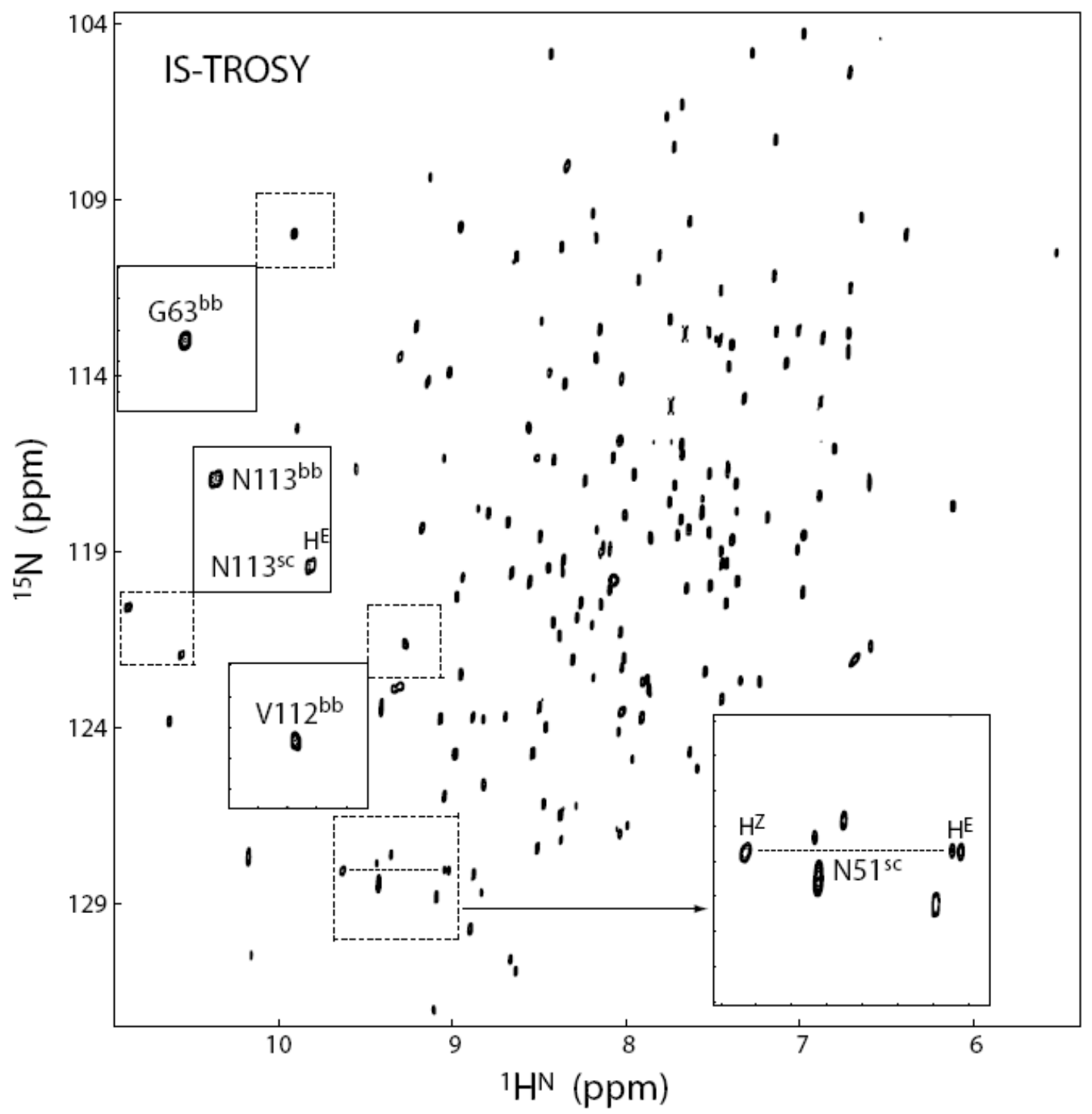

Figure S1 
Figure S1. 2D ${ }^{15} \mathrm{~N}-{ }^{1} \mathrm{H}$ IS-TROSY spectrum of $\left[\mathrm{U}-{ }^{2} \mathrm{H}^{13}{ }^{13}{ }^{15} \mathrm{~N}\right]-\mathrm{yCD}$ recorded on a Bruker AVANCE $900 \mathrm{MHz}$ NMR spectrometer equipped with a TCl cryoprobe at $25^{\circ} \mathrm{C}$. The NMR sample was made in $100 \mathrm{mM}$ potassium phosphate buffer, $\mathrm{pH} 7.0$ (without isotope correction), with $100 \mu \mathrm{M} \mathrm{NaN}$ and $20 \mu \mathrm{M}$ DSS (an internal NMR reference). The sample contained $\sim 1.5 \mathrm{mM}$ yCD (protomer concentration) and $20 \mathrm{mM}$ the transition state analog $5 \mathrm{FPy}$. The spectrum was recorded with 16 scans and a $2 \mathrm{~s}$ delay time, $t_{1 \max }\left({ }^{15} \mathrm{~N}\right)=53 \mathrm{~ms}$ and $t_{2 \max }\left({ }^{1} \mathrm{H}^{\mathrm{N}}\right)=285 \mathrm{~ms}$, resulting in the experimental time of $3.5 \mathrm{~h}$. Sequential and stereospecific NMR assignments of side-chain amides have been achieved with ISTROSY techniques and were reported earlier. ${ }^{5}$ The cross peaks of the backbone and side-chain amides that are involved in $\mathrm{H} \cdots \mathrm{O} \cdots \mathrm{H}$ bifurcated hydrogen bonds are indicated with dashed squares and enlarged in solid squares. Note that the backbone resonances of Gly63 and Val112 are singlets although their cross peaks are a bit broader, indicating that the corresponding ${ }^{2 \mathrm{~h}} \Delta^{1} \mathrm{H}$ isotope effects are not larger than the resonance line width ( $10 \mathrm{ppb}$ or $9 \mathrm{~Hz}$ at the $900 \mathrm{MHz}$ field). 